\title{
Massive thyroid goiter: an unusual cause of aspiration pneumonia
}

\author{
Ming-Tsung Chen and Chia-Hsin Liu
}

Division of Pulmonary and Critical Care Medicine, Department of Internal Medicine, Tri-Service General Hospital, National Defense Medical Center, Taipei, Taiwan
Received: December 9, 2015

Revised : January 22, 2016

Accepted: March 20, 2016

\section{Correspondence to}

Chia-Hsin Liu, M.D.

Tel: +886-2-8792-3311

Fax: +886-2-8792-7245

E-mail:arron12182012@gmail.com
A 76-year-old woman, admitted with a 5-day history of fever and productive cough, had been experiencing progressive dyspnea on exertion, dysphagia, and frequent choking on food, for the past few years. Her vital signs were blood pressure, 148/66 mmHg; heart rate, 78 beats per minute; respiration rate, 20 breaths per minute; body temperature, $38.7^{\circ} \mathrm{C}$. Physical examination revealed a large, soft, painless goiter in front of her neck (Fig. 1A). Chest X-ray revealed a neck mass with marked deviation of the trachea, and patchy opacities over the right lung field (Fig. 1B). Neck computed tomography revealed multiple heterogeneous enhancing nodules (up to $13.6 \mathrm{~cm}$ along the long axis) extending from the retropharyngeal region into the upper mediastinum, compressing and displacing the trachea, esophagus, and right internal jugular vein to the right (Fig. 2). Serum thyrotropin and free thyroxine levels were $0.15 \mu \mathrm{IU} / \mathrm{mL}$ (normal range, 0.25 to 5) and $0.84 \mathrm{ng} /$ $\mathrm{dL}$ (normal range, 0.8 to 2), respectively. An intravenous piperacillin/tazobactam injection for aspiration pneumonia resolved her clinical symptoms. Fine-needle aspiration biopsy showed a benign follicular nodule. She is scheduled for total thyroidectomy.

Thyroid nodule is a common disorder, especially in women; its frequency increases with age and iodine deficiency. The symptoms depend on the

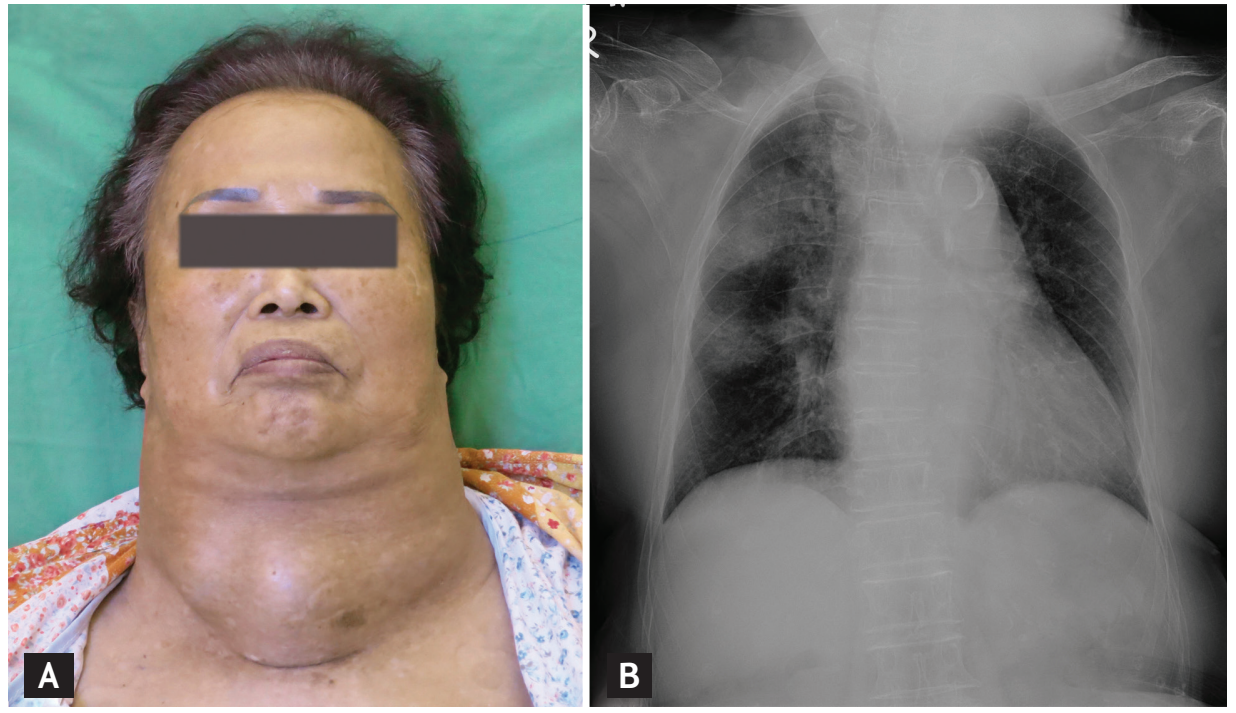

Figure 1. (A) A large neck goiter. (B) Chest X-ray showing a huge neck mass compression with tracheal narrowing and displacement, and patchy opacites in the right lung. 

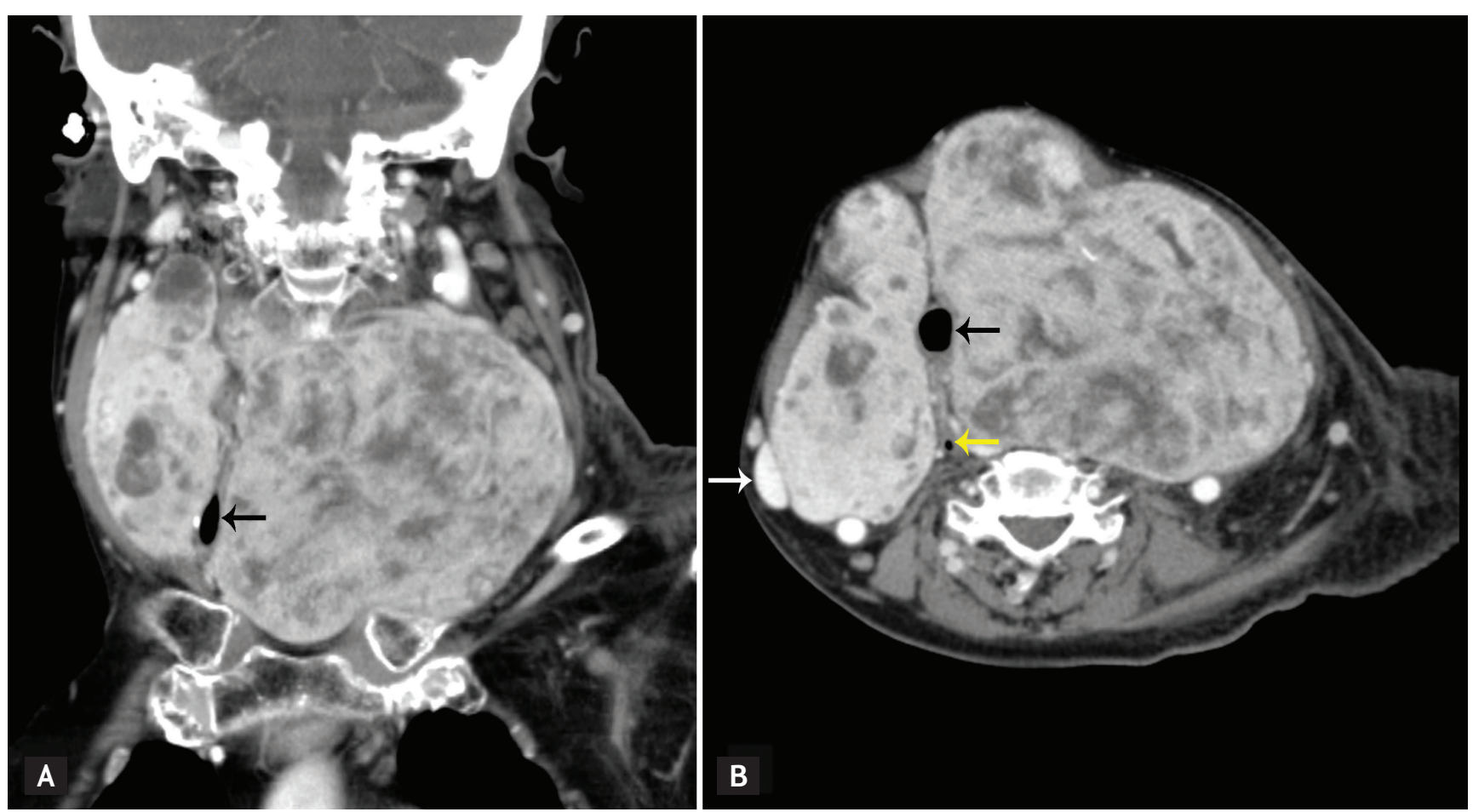

Figure 2. (A) Coronal computed tomography (CT) and (B) axial CT scan showing multiple heterogeneous nodules in the neck with compression and displacement of trachea (black arrows), right internal jugular vein (white arrow), and esophagus (yellow arrow).

presence of thyroid dysfunction and the growth rate of the goiter. Most patients are asymptomatic. Progressive enlargement of a multinodular thyroid goiter can cause compression of the trachea, esophagus, and vessels, resulting in cough, dyspnea, dysphagia, or feeling of a lump in the throat. In rare cases, esophageal compression secondary to retrosternal goiter may cause dysphagia and esophagopharyngeal reflux, leading to recurrent aspiration pneumonia, as in our case. Thy- roidectomy can rapidly relieve obstructive symptoms for patients with very large goiters. Furthermore, radioiodine therapy is an alternative for poor surgical candidates or for those who wish to avoid surgery.

\section{Conflict of interest}

No potential conflict of interest relevant to this article was reported. 